\title{
Inseminação artificial em tempo fixo
}

\author{
Kleber da Cunha Peixoto Junior ${ }^{1^{*}}$, Yessica Trigo ${ }^{2}$
}

${ }^{1}$ Médico Veterinário, D. Sc.,Professor do Curso de Medicina Veterinária, Universidade Metodista de São Paulo.

${ }^{2}$ Estudante de Graduação do Curso de Medicina Veterinária, Universidade Metodista de São Paulo. "Autor para correspondência, E-mai: ukcpunior@uol.com.br

\begin{abstract}
RESUMO.A inseminação artificial em tempo fixo (IATF) trata-se de uma biotecnologia reprodutiva que visa elevar a eficiência reprodutiva dos rebanhos por meio da indução e sincronização da ovulação das fêmeas através de protocolos hormonais. Assim, o objetivo deste trabalho foi fazer um levantamento bibliográfico sobre a IATF, sua origem, suas vantagens e limitações, tipos de protocolos e resultados. No Brasil, esta tecnologia começou a ser utilizada no início da década de 70, com a abertura das primeiras centrais de inseminação. Apresenta taxa de prenhez média que gira em torno de $50 \%$ a cada IATF, podendo atingir $60 \%$ e, em propriedades com ótimo manejo nutricional, sanitário e reprodutivo até $70 \%$.As principais vantagens da IATF são a realização da inseminação com dia e hora marcados, a eliminação da detecção de cio, inseminação das matrizes a partir de 60 dias após o parto, indução da ciclicidade em vacas em anestro, redução do intervalo entre partos, possibilidade de altas taxas de prenhez no início da estação de monta, entre outras. A maior parte dos problemas que interferem nos resultados da IATF está relacionada ao momento e ao manejo da inseminação artificial, à aplicação dos hormônios utilizados nos protocolos em quantidade e momento exato e à qualidade técnica do responsável pelo programa. Para obter produção de bons bezerros, a escolha do sêmen é fundamental. Assim, pode-se concluir que a utilização da IATF de forma correta, por profissionais capacitados permite elevar a produção de carne e/ou leite da propriedade.
\end{abstract}

Palavras-chave: IATF, taxa de prenhez, estro, bovinos

\section{Artificial inseminatiam in fixe time}

ABSTRACT.The artificial insemination in fixed time (AIFT) is in a reproductive biotechnology that aims to increase reproductive efficiency of livestock by means of induction and synchronization of ovulation in females by hormonal protocols. The objective of this study was to review the literature on the AIFT, the origin, the advantages and limitations, types of protocols and results. In Brazil, this technology was first used in the early $70 \mathrm{~s}$, with the opening of the first insemination centers. Displays average pregnancy rate that is around 50\% for each AIFT, reaching $60 \%$, and with great nutritional properties, health and reproductive management up to $70 \%$. The main advantages of AIFT are the realization of insemination marked with day and time, elimination of heat detection, insemination mothers from 60 days after delivery, induction of cyclicity in anestrous cows, reduced calving interval, possibility high pregnancy rates at the beginning of the breeding season, among others . Most problems that interfere with the results of AIFT are related to when and management of artificial insemination, the application of hormones used in protocols in quantity and exact time and technical quality of the responsibility for the program. To have good calf production, the choice of sperm is critical. Thus, we can conclude that the use of AIFT correctly by trained professionals can raise the production of meat and/or milk from the property.

Keywords: AIFT, pregnancy rate, estrus, cattle.

\section{Introdução}

Para obter avanço genético dos rebanhos, a Inseminação Artificial (I.A.) é a técnica mais utilizada no Mundo. No Brasil, começou a ser utilizada no início da década de 70 , com a abertura das primeiras centrais de inseminação. É considerada hoje uma tecnologia que mudou a pecuária não só no Brasil, mais no mundo todo, como descrito anteriormente. 
Estudos mostram que nos últimos dez anosocorreu grande crescimento no número de inseminações realizadas no território brasileiro, sendo esse crescimento bastante consistente nos últimos quatro anos. Segundo Asbia, em 2010, foram comercializadas 10.415 .070 de doses de sêmen, ou seja, apenas $8 \%$ das 71.988 .424 fêmeas em idade reprodutiva (acima de 24 meses) foram inseminadas (levando em consideração uma média de duas doses de sêmen por concepção), sendo uma parcela muito pequena quando comparada ao potencial brasileiro (Ferreira \& Vieira, 2011).

Através das dificuldades encontradas no processo de Inseminação Artificial (I.A.) Convencional, começou-se a usar a Inseminação Artificial em Tempo Fixo, ou também conhecida pela sigla IATF, que é considerada uma biotecnologia para resolver as dificuldades da IA convencional através do controle da ovulação por meio de protocolos hormonais. Está técnica hoje em dia é muito empregada em bovinos, mais pode ser também utilizada em diversas espécies, como, por exemplo, ovinos, caprinos e bubalinos (Meldau, 2007).

Cada vez mais a Inseminação Artificial entra na lista de compras do pecuarista, e o motivo principal disso é a Inseminação Artificial em Tempo Fixo (IATF). É só marcar o dia, a hora, sincronizar a ovulação e esperar o nascimento dos bezerros. No entanto, o índice de prenhez pode ficar um pouco abaixo quando comparado com o método convencional. Portanto, isso depende de fatores externos, como por exemplo, o armazenamento do sêmen. Uma das maiores vantagens da técnica é que não é necessário observar o cio, e isso é visto como o maior desafio para o criador atualmente (Pitombo, 2013).

O seu uso garante tamanhas vantagens que pode se afirmar que ela mudará o perfil do rebanho nacional em um curto período de tempo. Sendo assim, este artigo tem como objetivo fazer um levantamento bibliográfico sobre a IATF, sua origem, suas vantagens e limitações, tipos de protocolos e resultados.

\section{Origem da inseminaçãoartificial}

"Segundo a lenda a Inseminação Artificial (I.A.) foi utilizada pela primeira vez no ano de
1332, em equinos, pelos árabes. Mas a história registra como marco inicial da inseminação artificial o ano de 1784, quando o monge italiano de nome Lázaro Spallanzani demonstrou pela primeira vez ser possível a fecundação de uma fêmea sem o contato com um macho. Para tanto, ele coletou sêmen de um cachorro através da excitação mecânica e aplicou em uma cadela no cio, a qual veio a parir três filhotes 60 dias mais tarde. Era o nascimento de uma técnica que iria revolucionar o campo da reprodução animal"'(Asbia, 2010, p. 06).

No ano de 1949, Polge, Smith e Parker, pesquisadores ingleses, demonstraram que o espermatozoide podia ser conservado por um longo tempo a baixas temperaturas. Até então o sêmen era conservado refrigerado à temperatura de $5^{\circ} \mathrm{C}$, possibilitando aos espermatozóides sobrevida de 96 horas. Esta descoberta permitiu a sua conservação indefinidamente, dando maior difusão à inseminação artificial. Atualmente muitos países inseminam quase a totalidade de seus rebanhos bovinos. Calcula-se que mais de 106 milhões de fêmeas sejam anualmente inseminadas em todo o mundo. (Asbia, 2010, p. 06).

A indústria da I.A. em bovinos no mundo cresceu nos últimos trinta anos para situar-se hoje em dia em aproximadamente 106 milhões de primeiras inseminações após o parto. Em 1998 foram produzidas mais de 240 milhões de doses de sêmen de bovinos e búfalos,oriundas de 40.000 reprodutores em 600 centros de IA segundo dados de Thibier\& Wagner de 2000 (Asbia, 2010, p. 06).

No Brasil, segundo estimativas aproximadas apenas $7 \%$ das fêmeas em idade reprodutiva são inseminadas. A primeira inseminação que se tem noticia no Brasil data-se de 1940, porém comercialmente a técnica somente alcançou impulso a partir de 1970, quando nasceram as primeiras empresas especializadas no ramo (Asbia, 2010, p. 06).

\section{Evolução da IATF}

Atualmente, os criadores estão cada vez mais atentos às vantagens que a Inseminação Artificial em Tempo Fixo (IATF) traz aos seus rebanhos.Quando se fala sobre melhoramento genético, existem alguns meios que se tornam 
indispensáveis para facilitar o trabalho de manejo e melhorar a rentabilidade. O relatório de 2011 da Associação Brasileira de Inseminação Artificial (Asbia) destaca que a primeira inseminação realizada no Brasil foi em 1940, porém comercialmente a técnica somente alcançou impulso a partir de 1970, quando surgiram as primeiras empresas especializadas no ramo. Ainda, vale lembrar, que entre 1991 a 2010, a técnica teve um crescimento com mais de $300 \%$ no País (Rocha, 2011).

Já a IATF cresce em torno de 20 a $30 \%$ ao ano, porém, é na região Central do País, onde ela apresenta maior concentração. Segundo Cristiano Ribeiro, isso ocorre principalmente nos Estados de Mato Grosso, Mato Grosso do Sul, Goiás, Pará e no norte do Brasil, onde esta prática tem se expandido de uma maneira bem expressiva. A técnica está sendo muito utilizada e de forma muito profissional, tendo maior predominância no gado de corte. (Rocha \& Ribeiro, 2011). O Brasil já está em uma posição de destaque em relação aos outros países, sendo o maior mercado de IATF do mundo(Sandoval, 2011).

Através do crescimento da IATF, o mercado está cada vez mais confiante nas técnicas e tecnologias disponíveis para a reprodução bovina. Em geral, os produtores não costumam investir em novas técnicas em um primeiro momento, preferindo serem conservadores. "Pelo crescimento dos números de IATF registrados no País nos últimos anos, hoje a técnica é mais conhecida, trazendo resultados muito positivos para as propriedades, como: aumento da produtividade e lucratividade, além do melhoramento genético do rebanho" comenta Meneguetti (2011).

No ano de 2012, foi realizado Inseminação artificial em tempo fixo (IATF) em 8 milhões de fêmeas bovinas, sendo $75 \%$ delas das raças de corte e as outras $25 \%$ restantes das de leite. Esse valor representa $65 \%$ de todas as inseminações realizadas no Brasil, que beneficiam em aproximadamente $10 \%$ das fêmeas em idade reprodutiva (Pitombo, 2013).

Nesses valores nos mostram o quanto a técnica ainda pode crescer. No Brasil, a eficiência reprodutiva é baixa.
No Brasil, a baixa eficiência reprodutiva é caracterizada por intervalo entre parto (IEP) médio de 17 meses e período de serviço (do parto até a concepção) de 225 dias. No entanto, tecnologias como a IATF podem fazer com que a fêmeas fiquem gestante mais cedo, reduzindo o intervalo entre partos (Pitombo, 2013).

A inseminação artificial (IA) é considerada uma importante forma de expandir a genética, já que as buscas por touros ditos "melhoradores" já está sendo uma prática comum em diversas fazendas. Em geral, tendo o uso da inseminação artificial (IA) faz com que os produtores possam escolher tais touros selecionados de qualquer região do mundo e de acordo com os seus interesses comerciais, acelerando os efeitos das características desejadas no seu rebanho. Portanto, pode-se fazer a implantação de processos de IATF e com a eficiência do uso da IA requer boas taxas de detecção de cio.Por estes motivos a IATF está ganhando cada vez mais espaço e credibilidade a cada ano que passa nas propriedades brasileiras (Ferreira \& Vieira, 2011).

É provável que a evolução da IATF favoreça o crescimento de todos os índices de produção e produtividade(Ferreira \& Vieira, 2011). De acordo com os autores, há alguns anos as pesquisas realizadas no Brasil tem tido como foco a essa técnica, onde atualmente, já existem diversas alternativas viáveis para ter sucesso através de seu uso.Dessa maneira, a expansão da IATF pode ser uma ótima forma para elevar as perspectivas de crescimento da cadeia de produção da carne, visto que $92 \%$ de nossas matrizes ainda são acasaladas em sistema de monta natural.

\section{Indução da ovulação e sincronização do cio em fêmeas bovinas}

Devido ao aprimoramento dos conhecimentos da fisiologia reprodutiva da fêmea foi desenvolvido protocolos hormonais que permitema sincronização da ovulação e, consequentemente, a realização da IA em dia e hora programada, por isso chamado, tempo fixo (Meldau, 2007).

Dentre os hormônios mais utilizados estão os progestágenose seus análogos sintéticos, que na maioria das vezes são aplicados na forma de 
implantes vaginais ou auriculares, garantindo uma liberação gradativa deste hormônio. Além deste, também são utilizados estradiol, gonadotrofinas (LH e FSH) ou GnRH como indutores da ovulação e as prostaglandinas F2 $\alpha$ (PGF2 $\alpha$ )e seus análogos como luteolíticos (Odde, 1990).Por serem substâncias iguais, ou mesmo, similares às que participam do ciclo estral natural, vale a pena ressaltar que os fármacos e hormônios usados não prejudicam as fêmeas, e após os seus efeitos, também não interferem nos ciclos posteriores, e muito menos geram resíduos na carne e no leite (Pursley, Mee\&Wiltbank, 1995).

Assim, quando se busca melhorar a eficiência dos programas de sincronização baseados na utilização de progesterona e prostaglandina (PGF $2 \alpha$ ), tanto o crescimento folicular quanto a regressão do corpo lúteo são sincronização através da utilização de estrógenos e GnRH(Pursley, Mee\&Wiltbank, 1995).

Segundo Mesquita (2009), além destas substâncias, existem alguns programas que incluem preparações de gonadotrofina coriônica equina (eCG), glicoproteína com atividade predominante de FSH, e a gonadotrofina coriônica humana (hCG), glicoproteína com atividade predominante de $\mathrm{LH}$, conforme o período do ciclo estral. $\mathrm{O}$ fármaco mais utilizado no processo de sincronização do cio é a prostaglandina (PGF 2 $\alpha$ ). Entretanto, Mesquita (2009) também comenta que o estro após o tratamento é distribuído ao longo de seis dias, onde é influenciado não apenas pela responsividade do corpo lúteo, mas também pelo estágio de desenvolvimento do folículo dominante (Mesquita, 2009).

Os tratamentos realizados a base do uso de progesterona ou progestágeno tem como vantagem permitir a sincronização de estro em fêmeas ciclando, como também permite a indução da retomada da ciclicidade em vacas que estiverem em anestro. Já o tratamento usado no período pós-parto com progestágenos mantém o folículo dominante para que o mesmo alcance sua maturação final e assim ovule após a remoção do hormônio. Além disso, pode-se adiantar a puberdade em novilhas quando se faz a utilização pré-puberal de progesterona(Yavas\&Walton, 2000).
No entanto, Mesquita (2009) explica que não só a progesterona e a PGF $2 \alpha$ são os hormônios responsáveis por essas atividades ou funções, mas sim a ação conjunta dos diversos hormônios que constituem o eixo hipotálamo-hipófiseovário, ou seja, GnRH, FSH, LH, estrógeno, progesterona, prostaglandina, entre outros. Portanto, qualquer falha que ocorrer no transporte, produção, armazenamento, liberação e captação desses hormônios pelas células, ocasionarão distúrbios no comportamento reprodutivo da fêmea bovina (Mesquita, 2009).

\section{Vantagens e desvantagens da técnica de IATF}

Nesta biotecnologia as vacas têm ovulação induzida permitindo com que a IA seja realizada com hora e data marcada, garantindo maior produção e qualidade agregada ao rebanho (Baruselli, Reis \& Marques, 2004; Mesquita, 2009), pode-se inseminar um número maior de vacas (100 a 250 vacas inseminadas por dia) em um curto intervalo de tempo (Mesquita, 2009), programar o nascimento dos bezerros, elevar o número de bezerros ao início da estação de nascimentos e com isso obter um aproveitamento melhor da mão-de-obra. (Baruselli, Reis \& Marques, 2009).

Para Mesquita (2009) as principais vantagens da IATF são inseminação com dia e hora marcados; eliminação da detecção de cio; inseminação das matrizes a partir de 60 dias após o parto; indução da ciclicidade em vacas em anestro; redução no intervalo entre partos; possibilidade de altas taxas de prenhez no início da estação de monta; atingir o objetivo de obter 1 bezerro/vaca/ano; melhor acompanhamento reprodutivo das matrizes, descartando aquelas que se mostrarem improdutivas; padronização dos lotes de bezerros; redução do período de estação reprodutiva para 3 meses; redução na quantidade de touros de repasse no rebanho; racionalização da mão de obra, realocando funcionários da fazenda entre os diversos setores da mesma conforme a necessidade, ou contratando funcionários temporários para somente o período da IATF.

Segundo Baruselli, Reis \& Marques (2004), a falha na observação de cio diminui o número de bezerros nascidos, eleva o número de dias improdutivos dos animais e aumenta o intervalo 
entre partos, e quando isso é observado, muitos fazendeiros acabam parando de realizar o programa de inseminação artificial. Dessa maneira, com os programas de inseminação artificial em tempo fixo onde não há necessidade de realizar a detecção de cio, favorecem para o aumento da eficiência e do emprego da técnica de IA.

Porém, há alguns cuidados que devem ser seguidos para obter sucesso em programas com IATF como a utilização de inseminadores experientes;Médico Veterinário capacitado para a implantação de um programa de IATF; Mão de obra e instalações adequadas para a realização da inseminação; controle sanitário eficiente; Utilização de matrizes paridas a mais de 40 dias; boa condição corporal (de 2,5 ou mais) e utilização de sêmen de touros com alta fertilidade (Meldau, 2007).

Quando se realiza a IA após a detecção do estro, geralmente, as taxas de prenhez são maiores quando comparadas ao uso da IATF. (Yelich, Fields\&Sand, 2001). Mesquita (2009) cita que a causa mais provável para este fato pode ser atribuída às falhas na indução e sincronização das ovulações de vacas tratadas com progestágenos. Por outro lado, mesmo a IATF apresentando uma taxa menor de concepção quando comparada com a IA convencional (onde tem controle de estro), as taxas de prenhez são semelhantes devido à maior taxa de serviço da IATF (Bóet al., 2001).

Vale lembrar que alguns fatores favorecem para os baixos índices de produtividade como alta incidência de doenças parasitárias, infecciosas e nutricionais, a escassez nos programas de melhoramento genético, forragens de baixa qualidade e o sistema fundiário ultrapassado (Viana, 1999). Um dos pontos mais importantes que limita o desempenho da pecuária de corte brasileira é a falha na reprodução (Silva, 2005).

\section{Efeitos do sêmen e produtores que passaram a adotar a técnica}

Ainda não há dados necessários que nos permite identificar verdadeiramente quem são os usuários responsáveis pelo uso da IATF, mas mesmo assim considera que houve crescimento tanto por parte daqueles que já realizavam IA convencional anteriormente, como também por outros pecuaristas que passaram a adotar a técnica (Filho, 2013).

Como existem touros que, por motivos individuais, se mostram melhores em seus resultados a campo que os demais, assim, centrais realizam levantamentos entre veterinários e clientes, formando grupos de reprodutores mais indicados aos pecuaristas. Sendo assim, Rodrigues explica que a entidade, empresas do setor e pesquisadores iniciam estudos que irão estabelecer quais as melhores análises para se obter tais resultados no laboratório, sem precisar ficar na dependência da coleta de dados de campo (Pitombo, 2013).

A maioria dos problemas que interferem nos resultados da IATF está relacionada à forma que é feita a IA, à sincronização e ao manejo. Mas não podemos esquecer-nos da influência do efeito do touro, exemplificando que enquanto uns podem resultar em índices elevados de $70 \%$ de prenhez, outros ficam nos $40 \%$, mesmo tendo todo o cuidado que se tem nas análises de laboratório das centrais. Já em relação ao processo de sexagem, os resultados obtidos com este tipo de sêmen ficam em torno de $50 \%$, o que é considerável abaixo do produto convencional. Contudo, se fizer ajustes nos procedimentos de IATF, há indicações de como elevar estes resultados(Severo, 2013).

No presente momento, o que nos preocupa é em relação à contratação do serviço dos veterinários. Com a evolução da técnica, favorece aqueles profissionais que cobram pouco, mais que com isso podem ter pouca experiência e comprometer os resultados, o que não é favorável. Mais de qualquer forma, a perspectiva para os próximos anos é de um crescimento contínuo da IATF, incluindo a evolução no leite, e tendo uma proporcional redução da IA convencional (Filho, 2013).

\section{Controle para obter melhores resultados}

Atualmente, a taxa de prenhezem média gira em torno de $50 \%$ a cada IATF, mas pode atingir $60 \%$ e até $70 \%$ em algumas propriedades com manejo nutricional, sanitários e reprodutivos impecáveis (Baruselli, 2013).

Baruselli (2013) afirma que "os protocolos disponíveis demoraram anos para serem 
estabelecidos e estão plenamente definidos e com um patamar de resultados que não têm muito que mudar através da ciência". Para este autor, bons resultados dependem de equipes bem treinadas, profissionais e comprometidas com os resultados (Baruselli, 2013).

No Brasil, quando se utiliza sistema extensivo de criação de bovinos de corte, observa-se aproximadamente $50 \%$ das vacas estão em anestro no início da estação de acasalamento, principalmente devido a deficiências nutricionais e à baixa condição de saúde (Madureira et al., 2006).Sendo assim, estas vacas têm probabilidade de apresentarem baixas taxas de concepção quando forem submetidas à IATF (Mesquita, 2009).

Para obter produção de bons bezerros a escolha do sêmen é fundamental; sendo necessário utilizar os de maior fertilidade na técnica, vindos de empresas especializadas e que tenham alto valor genético. Por ter menor viabilidade no trato genital da vaca, o sêmen sexado terá resultados inferiores. No entanto, é possível aumentar as taxas de prenhez para perto de $80 \%$ da prenhez com sêmen convencional, ao se inseminar com "atraso" de 6 horas, em relação ao sêmen convencional, e assim, mais próximo da ovulação (Baruselli, 2013).

A IATF em relação a IA convencional se mostra mais eficiente. $\mathrm{O}$ uso da IATF possibilita que as inseminações e as prenhezes aconteçam no início da estação de monta (EM), garantindo diminuição do período de serviço e, consequentemente, melhora da eficiência reprodutiva no rebanho. Mesquita (2009) relatou que vacas da raça Brangus, nos primeiros 45 dias da estação de montaapresentaram maiores taxas de prenhez submetidas à IATF quando comparadas às vacas submetidas a IA convencional com detecção de cio(Baruselliet al., 2009).

\section{Conclusão}

A utilização da IATF de forma correta, feita por profissionais altamente capacitados, com sêmen de melhor qualidade, usando-se somente animais sadios e uma propriedade com boa infraestrutura, permite com que se aproveite ao máximo o potencial reprodutivo do rebanho, eleve a produção de carne e/ou leite por hectare e, consequentemente, proporcione aos produtores melhores retornos econômicos sem que haja a necessidade de ampliação das fronteiras agrícolas.

\section{Referências Bibliográficas}

Anderson, L.H.; McDowell, C.M.; Day, M.L. Progestin-induced puberty and secretion of leteinizing hormone in heifers.Biologie of Reproduction, v.54, n.5, p.1025-1031, 1996.

ASBIA. Associação Brasileira de Inseminação Artificial. Manual de inseminação artificial em bovinos. Minas Gerais, 2012. Disponível em: http://pt.slideshare.net/ruralbr/manualinseminao-

artificial?utmsource=slideshow01\&utm_medi um=ssemail\&utm_campaign=share_slidesho w_loggedout\%3E. Acesso em: 05/02/2013.

Baruselli, P.S. A sigla da qualidade superior dos bezerros. IATF, Porto Alegre, n. 172, ano. 16, p. 12-18, nov. 2013.

Baruselli, P.S.; Marques, M.O.; Carvalho, N.A.T.; Madureira, E.H.; Campos Filho, E.P. Efeitos de diferentes protocolos de inseminação artificial em tempo fixo na eficiência reprodutiva de vacas de corte lactantes. Revista Brasileira de Reprodução Animal, v.26, n.3, p.218-221, 2002.

Baruselli, P.S.; Reis, E.L.; Marques, M.O. Técnicas de manejo para aperfeiçoar a eficiência reprodutiva em fêmeas bosindicus. Grupo de Estudo de Nutrição de Ruminantes - Departamento de Melhoramento e Nutrição Animal - FCA - FMVZ - Unesp, Botucatu, São Paulo, 2004, p.18.

Bó, G.A.; Cutaia, L.; Brogliati, G.M.; Medina, M.; Tríbulo, R.; Tríbulo, H. Programas de inseminacion artificial a tiempo fixo em ganado bovino utilizando progestagenos y estradiol. In: SIMPOSIO INTERNACIONAL DE REPRODICCION ANIMAL, 4, 2001. Huerta Grande, Resúmenes... Huerta Grande: IRAC, 2001, p.117-136.

Ferreira, M. R. \& Vieira, M. L. O crescimento da IATF e seu impacto na cadeia produtiva da carne. Piracicaba, 2011. Disponível em: $<$ http://www.beefpoint.com.br/radarestecnicos/reproducao/o-crescimento-da-iatf-e- 
seu-impacto-na-cadeia-produtiva-da-carne72651/>. Acesso em: 18 abril de 2013.

Gottschall, C.S.; Canellas, L.C.; Marques, P.R.; Cruz, J.K.; Martins, O.; Almeida, M.R.; Bittencourt, H. R. Desempenho bioeconômico de vacas de corte submetidas à inseminação artificial em tempo fixo (iatf) em diferentes períodos pós-parto. $35^{\circ}$ Congresso Brasileiro de Medicina Veterinária (Conbravet 2008). Disponível em: <http://www.sovergs.com.br/conbravet2008/a nais/cd/resumos/R0645-1.PDF>. Acesso em: 26 de maio de 2013.

Madureira, E.H.; Fernandes R.H.R.; Rossa, Ferreira, L.A.; Braga, F.A.; Pardo, F.J.Anestro pós-parto em bovinos: A suplementação com óleos vegetais pode ser útil para encurta-lo? In. Simpósio Internacional de Reprodução Animal Aplicada, 2006, Londrina. Biotecnologia da Reprodução em Bovinos, 2006, p.63-70.

Mesquita, S.B. A importância da IATF para a Pecuária Brasileira. Eficiência Reprodutiva, São Paulo, n. 7, ano. 2, p. 4-8, mar/abr. 2009.

Odde, K.J. A review of synchronization of estrusin postpartum cattle. Journal Animal Science, v.68, p.817-830, 1990.

Pursley, J.R., Mee, M.O., Wiltbank, M.C. Synchronization of ovulation in dairy cattle using GnRH and PGF.Theriogenology, v. 44, p.915-923, 1995.

Rocha, C.; Meneguetti, M.; Sandoval, G.A.F.; Ribeiro, C. IATF, um mercado em expansão.
Mato Grosso. 2011. Disponível em: <http://boiapasto.com.br/2011/iatf-ummercado-em-expansao/>. Acesso em: $23 \mathrm{de}$ janeiro 2014.

Silva, L. F. P. Interface da nutrição com a reprodução: o que fazer? In: CONGRESSO BRASILEIRO DE REPRODUÇÃO ANIMAL, Goiânia: GO - GERAEMBRYO, p. 1-12, 2005.

Viana, J. A. C. O terceiro mundo não é assim: está assim! Belo Horizonte: FEPMVZ/UFMG - Escola de Veterinária, p.689, 1999.

Yavas, Y. \&Walton, J.S. Postpartum acyclicity in suckled beef cows: a review. Theriogenology, v.54, p.25-55, 2000.

Yelich, J.V. A vaginal insert (CIDR) to synchronize estrus and timed AI. In: Fields, M.J., Sand, R.S., Yelich, J.V. Factors affecting calf crop - Biotechnology of reproduction. Cap. 7, p.87-100, 2001.

Recebido em Junho 02, 2014.

Aceito em Julho 22, 2014.

License information: This is an open-access article distributed under the terms of the Creative Commons Attribution License, which permits unrestricted use, distribution, and reproduction in any medium, provided the original work is properly cited. 